\title{
Superexchange coupling in iron/silicon layered structures
}

\author{
V. V. Tugushev, ${ }^{1,2}$ V. N. Men'shov, ${ }^{1}$ I. A. Nechaev, ${ }^{2}$ and E. V. Chulkov ${ }^{2,3}$ \\ ${ }^{1}$ RRC Kurchatov Institute, Kurchatov Sqr. 1, 123182 Moscow, Russia \\ ${ }^{2}$ Donostia International Physics Center (DIPC), P. Manuel de Lardizabal, 4, 20018, San Sebastián, Basque Country, Spain \\ ${ }^{3}$ Departamento de Física de Materiales, Facultad de Ciencias Químicas, UPV/EHU and Centro Mixto CSIC-UPV/EHU, Apdo. 1072, \\ 20080 San Sebastián, Basque Country, Spain
}

(Received 12 May 2006; published 20 November 2006)

\begin{abstract}
We propose the mechanism of interlayer exchange coupling in $\mathrm{Fe} / \mathrm{Si}$ structures, based on three principal ideas: (i) Contact induced ferromagnetic phase of body-centered-cubic iron silicide and spin-polarized interfacial states are formed at the Fe/Si boundaries; (ii) exchange coupling between Fe layers is effectuated by means of the superexchange of spin-polarized interfacial states through the nonmagnetic semiconductor spacer; (iii) the complex character of the dependence of interlayer exchange coupling on the spacer thickness and composition is due to the competition between antiferromagnetic and ferromagnetic components of superexchange. We calculate the bilinear and biquadratic components of an exchange coupling energy in the framework of a simple two-band scheme of electron spectrum inside the spacer at zero temperature. Our model qualitatively explains existing experimental results.
\end{abstract}

DOI: 10.1103/PhysRevB.74.184423

PACS number(s): 73.40.Sx, 75.70.-i

\section{INTRODUCTION}

During the last years the silicon based magnetic nanostructures (sandwiches, multilayers, digital, and amorphous alloys) became a special topic of experimental and theoretical researches, due to their possible applications to spintronics. ${ }^{1}$ A number of works has been done in order to understand magnetic and transport properties of $\mathrm{Fe} / \mathrm{Si}$ layered structures. In particular, the origin of interlayer exchange coupling (IEC), i.e., magnetic coupling between $\mathrm{Fe}$ layers across the $\mathrm{Si}$ spacer, has been studied and discussed (for a review, see Ref. 2 and references therein).

The strength of IEC, being strongly dependent on the spacer thickness $L$, is characterized by two parametersintegrals of bilinear $J_{1}(L)$ and biquadratic $J_{2}(L)$ coupling, respectively. These integrals enter as coefficients in the series expansion of phenomenological exchange energy $E_{e x}$ of IEC in terms of magnetic moments $\mathbf{M}( \pm l)$ of neighbor ferromagnetic layers separated by the $\mathrm{Si}$ spacer of the thickness $L=2 l$ :

$$
E_{e x}=J_{1}(L) \mathbf{M}(-l) \mathbf{M}(+l)+J_{2}(L)[\mathbf{M}(-l) \mathbf{M}(+l)]^{2} .
$$

The integral $J_{2}(L)$ is always positive, while $J_{1}(L)$ may be positive or negative; below, we define $J_{1}(L)>0$ for the antiferromagnetic (AFM) coupling and $J_{1}(L)<0$ for the ferromagnetic (FM) coupling, respectively.

There exist controversial experimental results on the behavior of $J_{1}(L)$ as a function of the thickness $L$ in $\mathrm{Fe} / \mathrm{Si}$ systems. In particular, a very weak AFM IEC has been found in Ref. 3 while unusually strong AFM coupling and exponential decay of $J_{1}(L)$ at large $L>12-16 \AA$ have been well established in Refs. 4-9. On the other hand, dramatic increasing of $J_{1}(L)$ and evident crossover from AFM to FM character of IEC at $L<18-20 \AA$ were observed in Ref. 10 .

A strong biquadratic component $J_{2}(L)$ of IEC exponentially decaying at large $L$ has been revealed in Refs. 5-8, where different explanations of the origin of the biquadratic coupling in $\mathrm{Fe} / \mathrm{Si}$ structures have been proposed. However, long-standing discussions have not led to understanding the situation.

The interpretation of IEC data ${ }^{3-10}$ in terms of tunneling of spin-polarized quasiparticles between two layers of a ferromagnetic metal across the thin intermediary spacer of a nonmagnetic insulator (see Ref. 11) is hampered by the lack of knowledge of the crystal structure and electron properties of the iron-silicide $\left(\mathrm{Fe}_{1-x} \mathrm{Si}_{x}\right)$ interlayer formed by an uncontrolled diffusion of $\mathrm{Fe}$ atoms into the spacer through the $\mathrm{Fe} / \mathrm{Si}$ interface. Various models of this interlayer, from a nonmagnetic semimetal with the body-centered-cubic (bcc) structure (c-FeSi) to more complex compounds, have been proposed. ${ }^{12}$

Recent experiments ${ }^{2,13-16}$ performed on samples with a controlled composition $x$ in the $\mathrm{Fe}_{1-x} \mathrm{Si}_{x}$ spacer, $0.5<x<1$, demonstrated AFM character of IEC, exponential decay of $J_{1}(L)$ at large $L$, and pronounced maximum of $J_{1}(L)$ at some characteristic thickness $L_{\max }(x) \approx 8-16 \AA$ for all $x$. At large iron content ( $x$ approaches 0.5$) J_{1}(L)$ shows a smooth maximum at $L \approx 14-16 \AA$. Upon increasing the silicon content $(x$ approaches 1) the dramatic rise of IEC and the shift of the $J_{1}(L)$ peak to $L \approx 8-10 \AA$ has been observed.

These facts obviously contradict the standard "tunnel" mechanism. ${ }^{11}$ It is well known that solid solutions $\mathrm{Fe}_{1-x} \mathrm{Si}_{x}$ with $0.5<x<1$ are semiconductors. With increasing the silicon content the band gap in the electron spectrum of $\mathrm{Fe}_{1-x} \mathrm{Si}_{x}$ and the corresponding barrier for electron tunneling between metallic layers through the spacer increase. So, the integral $J_{1}(L)$, being proportional to the integral of tunneling, should decrease, due to the decrease of the electron tunneling length in the spacer. Experiments ${ }^{2,13-16}$ demonstrate an opposite behavior of $J_{1}(L)$ and reveal that iron diffusion suppresses IEC in $\mathrm{Fe} / \mathrm{Si}$ structures.

In pace with the experimental studies of IEC, several theoretical approaches have been proposed. For example, in a simple model of Ref. 17 it was suggested that the coupling between FM layers through an insulating spacer is due to the spin-dependent tunneling of free electrons across the rectan- 
gular potential barrier. In a more complex electron-optic model $^{18}$ the IEC is expressed in terms of spin-depended asymmetric reflection of quasiparticles at the insulatorferromagnetic interface. Both these models predict the AFM character and exponential decay of $J_{1}(L)$, as the spacer thickness $L$ increases. However, as was shown within the extended $s$ - $d$ exchange model, ${ }^{19}$ the IEC decrease could be either monotonic or oscillatory (from FM to AFM coupling) function of $L$, depending on the band structure and crystalline orientation of the semiconductor spacer. The authors of Ref. 20 performed an $a b$ initio study of IEC in the metalsemimetal system Fe/c-FeSi (see also Ref. 21) and applied the model of Ref. 18 to estimate the asymptotic behavior of IEC. In systems with a metallic spacer IEC is normally associated with the Ruderman-Kittel-Kasuya-Yosida (RKKY) exchange. ${ }^{22}$ In a free-electron gas model, the $J_{1}(L)$ function oscillates with a period defined by geometry of the Fermi surface, while in a more complex model of "Anderson's layers" the AFM bias of these oscillations has been predicted. ${ }^{23}$ Note that the pronounced oscillations of $J_{1}(L)$ have never been detected in the $\mathrm{Fe} / \mathrm{Fe}_{1-x} \mathrm{Si}_{x}$ structures with semiconducting spacers $(x>0,5)$, instead they have been demonstrated clearly in especially grown structures with metallic spacers $(x<0,5){ }^{2}$

From these brief remarks we conclude that the origin of IEC in Fe/Si layered systems is still far from being understood. In our opinion, new approaches are necessary to reconcile controversial experimental results, taking into consideration the particularities of composition, geometry, and methods of growing the concrete structure. The main theoretical difficulties in such a way are provided by the following circumstances.

First, the charge- and spin-density redistribution at the interface strongly influences the electron and magnetic structure of the spacer. In particular, interfacial electron states are sure to appear, their occupation and spin polarization strongly differ from those in the bulk material.

Second, the $\mathrm{Fe}_{1-x} \mathrm{Si}_{x}$ compound inside the spacer has to be considered as heavily doped magnetic semiconductor or semimetal. As a result, very complex electron structure of these materials, which comprises wide and narrow bands, has to explicitly be taken into account.

Below, we propose the model of IEC taking into consideration above-discussed aspects of the problem. For simplicity, we consider only the structures with the (001) direction of growing.

\section{SPIN-POLARIZED STATES AND CONTACT-INDUCED FERROMAGNETISM AT THE IRON/SILICON INTERFACE}

As has been demonstrated with the low-energy electron diffraction (LEED) measurements, ${ }^{2}$ the growth of a thin (5 $\AA$ ) $\mathrm{Fe}_{1-x} \mathrm{Si}_{x}$ layer on $\mathrm{Fe}(001)$ substrate is pseudomorphic even at $x \approx 1$ (i.e., for nominally pure silicon). A good compatibility of the metal and semiconductor lattices, as well as the almost ideal smoothness of their interface, has been established in these experiments. This conclusion was also confirmed by x-ray photoelectron spectroscopy measurements. ${ }^{9}$ We suppose that chemical reaction between $\mathrm{Fe}$ and Si takes place during the growing, and a thin intermediate layer of bcc iron-silicide (c-FeSi) appears at the $\mathrm{Fe} / \mathrm{Si}$ interface. The lattice parameter of the bulk c-FeSi is estimated to be of $2.86 \AA$, i.e., close to that of bcc Fe; the thickness of the intermediate layer is at least of $5 \AA$ even for structures with the suppressed diffusion, ${ }^{2}$ though it can achieve 10-14 $\AA$ for structures with the strong diffusion. ${ }^{9}$

As has been established in Ref. 20, the bulk c-FeSi is a paramagnetic semimetal with very small density of states (DOS) at the Fermi level and sharp empty peak of DOS lying on $0.2-0.3 \mathrm{eV}$ above the Fermi level. The bulk single crystal of c-FeSi is unstable with respect to structural transformation to a more complex crystalline modification $\varepsilon-\mathrm{FeSi}$, which is an extremely narrow-gap $(\sim 0.05 \mathrm{eV})$ semiconductor. We shall not discuss here contradicting views on the origin of magnetic and structural transformations in $\varepsilon-\mathrm{FeSi}$, since this is not the aim of our paper.

The bcc modification c-FeSi appears to be stable in a form of a thin intermediate layer sandwiched by the bcc Fe layer, due to the good compatibility of the lattice parameters. ${ }^{20}$ Obviously, both the occupation and spin polarization of electron states in such a layer significantly differ from those in the bulk c-FeSi. Our principal assumption is that the spindepended interfacial potential induces the strong charge and spin redistribution at the scale of few interlayer distances. As a result the double charged layer appears, due to the electron flow from Fe to c-FeSi. The Coulomb part of the interfacial potential shifts down the sharp DOS peak in c-FeSi, while the exchange part splits this peak into the spin-up and spindown parts. Following our scenario, the spin-up states are filled and the spin-down states are empty, therefore a contactinduced ferromagnetic phase (FM c-FeSi) is formed at the interface.

Note that the bulk phase (FM $\varepsilon$-FeSi) has been already discussed in Ref. 24. It appears from the paramagnetic phase (PM $\varepsilon-\mathrm{FeSi}$ ) by means of metamagnetic phase transition and the semiconductor-metal type of transformation of electron band structure under applying an external magnetic field. Obviously, in our case no phase transition occurs and the phase $(\mathrm{FM} \mathrm{c}-\mathrm{FeSi}$ ) is formed at the $\mathrm{Fe} / \mathrm{Si}$ interface during the nanostructure growing.

Strictly speaking, to formally describe the complex structure of the $\mathrm{Fe} / \mathrm{Si}$ interface we would introduce the symbols: (a) $\mathrm{Fe} /(\mathrm{FM} \mathrm{c}-\mathrm{FeSi}) / \mathrm{Si}$, if the diffusion of iron is weak and the iron-silicide occupies only the small part of the spacer; (b) $\mathrm{Fe} /(\mathrm{FM} \mathrm{c}-\mathrm{FeSi}) /(\mathrm{PM} \mathrm{c}-\mathrm{FeSi}) / \mathrm{Si}$, if the diffusion of iron is strong and all the spacer is occupied by the iron-silicide. For simplicity, we use in the following only traditional symbol $\mathrm{Fe} / \mathrm{Si}$ for the interface.

\section{MODEL HAMILTONIAN}

Let us consider a model of a relatively thick spacer of a nonmagnetic semiconductor with the nominal thickness $L=2 l$ in the direction $z$, embedded between two layers of a 
ferromagnetic metal. The axis $z$ is oriented perpendicular to the perfectly smooth metal-semiconductor interfaces, arranged symmetrically relative to the origin $z=0$ in the middle of the spacer. We presume that a very thin intermediate region with a characteristic scale $l_{i} \ll l$ is formed at the interface (intermediate layer). The model Hamiltonian $H_{S}$ of the spacer in such a system can be written in the following form:

$$
H_{S}=H_{i}+H_{i b}+H_{b} .
$$

Here $H_{i}$ is the Hamiltonian of electron states in the intermediate region (interfacial states):

$$
\begin{gathered}
H_{i}=H_{i}(+l)+H_{i}(-l), \\
H_{i}( \pm l)=\sum_{\alpha \beta} \int \frac{d \mathbf{q}}{(2 \pi)^{2}} a_{\alpha}^{+}(\mathbf{q}, \pm l)\left[E_{i}(\mathbf{q}, \pm l) \delta_{\alpha \beta}\right. \\
\left.+J_{i}( \pm l) \mathbf{M}( \pm l) \sigma_{\alpha \beta}\right] a_{\beta}(\mathbf{q}, \pm l),
\end{gathered}
$$

where $a_{\alpha}^{+}$and $a_{\alpha}$ are the creation and annihilation operators of quasiparticles with energy $E_{i}(\mathbf{q}, \pm l), \mathbf{q}$ is the twodimensional quasimomentum in the $(x, y)$ plane orthogonal to the axis $z, J_{i}( \pm l)$ is the exchange integral between the FM and intermediate states, $\mathbf{M}( \pm l)$ are the magnetization vectors of the FM layers, $\boldsymbol{\sigma}$ is the vector composed of the Pauli matrices, $(\alpha, \beta)$ are spin indices. Below we suppose that $E_{i}(\mathbf{q}, \pm l)$ and $J_{i}( \pm l)$ are identical for both interfaces: $E_{i}(\mathbf{q},+l)=E_{i}(\mathbf{q},-l)=E_{i}(\mathbf{q}), J_{i}(+l)=J_{i}(-l)=J_{i}$. The modules $M$ of vectors $\mathbf{M}( \pm l)=M \hat{\mathbf{e}}( \pm l)$ are also identical for both interfaces, while their directions $\hat{\mathbf{e}}( \pm l)$ may strongly differ from each other: $\hat{\mathbf{e}}(+l) \neq \hat{\mathbf{e}}(-l)$.

The Hamiltonian $H_{i b}$ describes the one-electron hybridization between the interfacial states and states inside the spacer ("bulk states"); it may be written in the framework of the plane defect model:

$$
\begin{gathered}
H_{i b}=H_{i b}(+l)+H_{i b}(-l), \\
H_{i b}( \pm l)=\sum_{\alpha, m} \int \frac{d \mathbf{q} d \mathbf{q}^{\prime} d k_{z}}{(2 \pi)^{5}} b_{m, \alpha}^{+}\left(\mathbf{q}, k_{z}\right) \\
\times V_{m}\left(\mathbf{q}, \mathbf{q}^{\prime}, \pm l\right) a_{\alpha}\left(\mathbf{q}^{\prime}, \pm l\right) e^{ \pm i k_{z} l}+\text { H.c., }
\end{gathered}
$$

where $b_{m, \alpha}^{+}$and $b_{m, \alpha}$ are the creation and annihilation operators of quasiparticles in the $m$ th band inside the spacer, $k_{z}$ is the projection of the three-dimensional momentum $\mathbf{k}$ $=\left(\mathbf{q}, k_{z}\right)$ onto the $z$ axis, $V_{m}\left(\mathbf{q}, \mathbf{q}^{\prime}, \pm l\right)$ are the matrix elements of hybridization at the interface (i.e., matrix elements of the interfacial potential) assumed to be equal for both interfaces, so that $V_{m}\left(\mathbf{q}, \mathbf{q}^{\prime},+l\right)=V_{m}\left(\mathbf{q}, \mathbf{q}^{\prime},-l\right)=V_{m}\left(\mathbf{q}, \mathbf{q}^{\prime}\right)$. Note that the function $V_{m}\left(\mathbf{q}, \mathbf{q}^{\prime}\right)$ is nonzero for arbitrary $\mathbf{q}$ and $\mathbf{q}^{\prime}$, since the interfacial and "bulk" states correspond to the irreducible representations of different groups of crystal symmetry.

Finally, the Hamiltonian $H_{b}$ of the bulk states has the form

$$
H_{b}=\sum_{m, \alpha} \int \frac{d \mathbf{k}}{(2 \pi)^{3}} b_{m, \alpha}^{+}(\mathbf{k}) E_{m}(\mathbf{k}) b_{m, \alpha}(\mathbf{k}) .
$$

Below we consider a simple two-band model $(m=V, C)$ of a nondegenerated semiconductor. We suppose that the wide valence $\left[E_{V}(\mathbf{k})\right]$ and conduction $\left[E_{C}(\mathbf{k})\right]$ bands have the maximum and minimum at vectors $\mathbf{K}_{V}$ and $\mathbf{K}_{C}$ of the first Brillouin zone, respectively. Near the maximum (minimum) we write

$$
\begin{aligned}
& E_{V}(\mathbf{k})=E_{V}\left(\mathbf{K}_{V}\right)-\frac{\left(\mathbf{k}-\mathbf{K}_{V}\right)^{2}}{2 m_{V}}, \\
& E_{C}(\mathbf{k})=E_{C}\left(\mathbf{K}_{C}\right)+\frac{\left(\mathbf{k}-\mathbf{K}_{C}\right)^{2}}{2 m_{C}} .
\end{aligned}
$$

Here $m_{V, C}$ are the corresponding effective masses. We define the energy gap of the semiconductor as $E_{g}=E_{C}\left(\mathbf{K}_{C}\right)$ $-E_{V}\left(\mathbf{K}_{V}\right)$ and take the bandwidths $W_{V} \sim W_{C} \gg E_{g}$.

\section{THERMODYNAMICAL POTENTIAL AND THE ENERGY OF SUPEREXCHANGE COUPLING}

From Eqs. (1)-(6), it is evident that the coupling between interfacial states at the left and right boundaries of the spacer vanishes in the absence of the hybridization term (4) of Hamiltonian (1). If we consider the term (4) as a perturbation, it is possible to obtain the addition $\Delta \Omega$ to the total thermodynamic potential $\Omega$ of the system in a form of series expansion over the potential $V$ using the Green's-function method. ${ }^{25}$ It is evident that only even terms are not vanishing in such an expansion and we write in the fourth order of the perturbation theory:

$$
\Delta \Omega=\Delta \Omega_{2}+\Delta \Omega_{4},
$$

$$
\begin{aligned}
\Delta \Omega_{4}= & \frac{1}{4} \sum_{m n, \alpha \beta} \oint \frac{d \omega}{2 \pi i} \int \frac{d \mathbf{q} d \mathbf{q}^{\prime} d \mathbf{q}^{\prime \prime} d \mathbf{q}^{\prime \prime \prime}}{(2 \pi)^{8}} V_{m}^{*}\left(\mathbf{q}, \mathbf{q}^{\prime}\right) V_{m}\left(\mathbf{q}^{\prime}, \mathbf{q}^{\prime \prime}\right) V_{n}^{*}\left(\mathbf{q}^{\prime \prime}, \mathbf{q}^{\prime \prime \prime}\right) V_{n}\left(\mathbf{q}^{\prime \prime \prime}, \mathbf{q}\right) \\
& \times\left[G_{i}^{\alpha \beta}(\omega, \mathbf{q},-l) G_{i}^{\beta \alpha}\left(\omega, \mathbf{q}^{\prime \prime},-l\right) G_{m}^{\beta \beta}\left(\omega, \mathbf{q}^{\prime}, 0\right) G_{n}^{\alpha \alpha}\left(\omega, \mathbf{q}^{\prime \prime \prime}, 0\right)+G_{i}^{\alpha \beta}(\omega, \mathbf{q},+l) G_{i}^{\beta \alpha}\left(\omega, \mathbf{q}^{\prime \prime},+l\right) G_{m}^{\beta \beta}\left(\omega, \mathbf{q}^{\prime}, 0\right) G_{n}^{\alpha \alpha}\left(\omega, \mathbf{q}^{\prime \prime \prime}, 0\right)\right. \\
& \left.+G_{i}^{\alpha \beta}(\omega, \mathbf{q},-l) G_{i}^{\beta \alpha}\left(\omega, \mathbf{q}^{\prime \prime},+l\right) G_{m}^{\alpha \alpha}\left(\omega, \mathbf{q}^{\prime}, L\right) G_{n}^{\beta \beta}\left(\omega, \mathbf{q}^{\prime \prime \prime},-L\right)+G_{i}^{\alpha \beta}(\omega, \mathbf{q},+l) G_{i}^{\beta \alpha}\left(\omega, \mathbf{q}^{\prime \prime},-l\right) G_{m}^{\alpha \alpha}\left(\omega, \mathbf{q}^{\prime},-L\right) G_{n}^{\beta \beta}\left(\omega, \mathbf{q}^{\prime \prime \prime}, L\right)\right] .
\end{aligned}
$$


Here $G_{i}^{\alpha \beta}$ and $G_{m}^{\alpha \beta}$ are the Green's functions of the interfacial and bulk states, respectively; $\omega$ is a frequency. In this work we perform all calculations at zero temperature. However, they can also be performed at finite temperature, by means of relevant diagram techniques. ${ }^{25}$

Let us define the IEC energy $\Delta \Omega_{e x}$ as a part of $\Delta \Omega$ bilinear over the magnetization vectors of the adjacent FM layers:

$$
\Delta \Omega_{e x}=I(L) \mathbf{M}(-l) \mathbf{M}(+l),
$$

where $I(L)$ is the exchange integral. From Eqs. (8)-(10) it is clear that the first nonvanishing contribution to $\Delta \Omega_{e x}$ arises from the term $\Delta \Omega_{4}$, i.e., the value $I(L)$ is proportional to $V^{4}$.

To obtain the explicit expression for $I(L)$, we approximate the integrals in Eq. (10) in the following way. It is clear that in the Green's functions $G_{m}^{\alpha \beta}$ only momenta close to the points $\mathbf{K}_{V}$ and $\mathbf{K}_{C}$ give essential contribution to these integrals. On the other hand, in the Green's functions $G_{i}^{\alpha \beta}$ all momenta contribute to the DOS peak and to integrals (10). Thereby the hybridization processes with the transfer of large momenta play an important role. To consider this circumstance, one may formally pass to the sum over momenta $\mathbf{q}$ inside the first Brillouin zone (BZ) as $\int d \mathbf{q} /(2 \pi)^{2} \rightarrow \Sigma$ and use the "mean-square" approximation:

$$
\mathbf{q} \in B Z
$$

$$
\begin{gathered}
\sum_{\mathbf{q}^{\prime \prime} \in B Z} V_{m}\left(\mathbf{q}, \mathbf{q}^{\prime \prime}\right) G_{i}^{\alpha \beta}\left(\omega, \mathbf{q}^{\prime \prime}, \pm l\right) V_{n}^{*}\left(\mathbf{q}^{\prime \prime}, \mathbf{q}^{\prime}\right) \\
\approx(2 \pi)^{2} \gamma_{m n} \Gamma^{\alpha \beta}(\omega, \pm l) \delta_{\mathbf{q}-\mathbf{q}^{\prime}, \mathbf{K}_{m n}^{\perp}} \\
\Gamma^{\alpha \beta}(\omega, \pm l)=\sum_{\mathbf{q}^{\prime \prime} \in B Z} G_{i}^{\alpha \beta}\left(\omega, \mathbf{q}^{\prime \prime}, \pm l\right) \\
\gamma_{m n}=\sum_{\mathbf{q}^{\prime \prime} \in B Z} V_{m}\left(\mathbf{K}_{m}^{\perp}, \mathbf{q}^{\prime \prime}\right) V_{n}^{*}\left(\mathbf{q}^{\prime \prime}, \mathbf{K}_{n}^{\perp}\right)
\end{gathered}
$$

where the frequency-dependent pseudopotentials at the interface $\gamma_{m n} \Gamma^{\alpha \beta}(\omega, \pm l)$ are introduced. We presume that vectors $\mathbf{K}_{m n}=\mathbf{K}_{m}-\mathbf{K}_{n}$ lie in the first Brillouin zone, $\mathbf{K}_{m n}^{\perp}$ is the projection of $\mathbf{K}_{m n}$ onto the $(x, y)$ plane.

In our model the bandwidths $W_{m, n}$ of bulk states significantly exceed the width $W_{i}$ of the DOS peak of interfacial states and the Fermi level is situated inside the energy gap $E_{g}$. Within these assumptions, we write the following expression for $I(L)$ :

$$
\begin{aligned}
I(L)= & \frac{J_{i}^{2}}{2} \sum_{m n}\left|\gamma_{m n}\right|^{2} e^{i K_{m n}^{z} L} \oint \frac{d \omega}{2 \pi i} \int \frac{d \mathbf{q} d k_{z} d k_{z}^{\prime}}{(2 \pi)^{4}} D^{2}(\omega) \\
& \times G_{m}\left(\omega, \mathbf{q}, k_{z}\right) G_{n}\left(\omega, \mathbf{q}, k_{z}^{\prime}\right) e^{i\left(k_{z}-k_{z}^{\prime}\right) L},
\end{aligned}
$$

where

$$
\begin{gathered}
G_{m}\left(\omega, \mathbf{q}, k_{z}\right)=\frac{1}{\omega-E_{m}(\mathbf{k})}, \\
D(\omega)=\int \frac{d \varepsilon N_{i}(\varepsilon)}{(\omega-\varepsilon)^{2}-\left(J_{i} M\right)^{2}} .
\end{gathered}
$$

Here $N_{i}(\varepsilon)$ is the DOS of interfacial states, $K_{m n}^{z}$ is the projections of $\mathbf{K}_{m n}$ onto the $z$ axis; the vector $\mathbf{k}$ in the Green's function $G_{m}(\omega, \mathbf{k})=G_{m}\left(\omega, \mathbf{q}, k_{z}\right)$ is measured from the minimum or maximum of $E_{m}(\mathbf{k})$.

In the two-band model $(7)$, where $(m, n)=(C, V)$, we rewrite Eq. (13) in the following form:

$$
I(L)=I_{1}(L)+I_{2}(L) \cos \left(K_{0}^{z} L\right),
$$

where $\mathbf{K}_{0}=\mathbf{K}_{C}-\mathbf{K}_{V}$ and

$$
\begin{gathered}
I_{1}(L)=\left|\gamma_{C C}\right|^{2} A_{C}(L)+\left|\gamma_{V V}\right|^{2} A_{V}(L), \\
I_{2}(L)=\left|\gamma_{C V}\right|^{2} A_{C V}(L)+\left|\gamma_{V C}\right|^{2} A_{V C}(L),
\end{gathered}
$$

where

$$
\begin{aligned}
& A_{C}(L)=\frac{J_{i}^{2}}{2} \oint \frac{d \omega}{2 \pi i} \int \frac{d \mathbf{q}}{(2 \pi)^{2}} F_{C}^{*}(\omega, \mathbf{q}, L) F_{C}(\omega, \mathbf{q}, L), \\
& A_{V}(L)=\frac{J_{i}^{2}}{2} \oint \frac{d \omega}{2 \pi i} \int \frac{d \mathbf{q}}{(2 \pi)^{2}} F_{V}^{*}(\omega, \mathbf{q}, L) F_{V}(\omega, \mathbf{q}, L), \\
& A_{C V}(L)=\frac{J_{i}^{2}}{2} \oint \frac{d \omega}{2 \pi i} \int \frac{d \mathbf{q}}{(2 \pi)^{2}} F_{C}^{*}(\omega, \mathbf{q}, L) F_{V}(\omega, \mathbf{q}, L), \\
& A_{V C}(L)=\frac{J_{i}^{2}}{2} \oint \frac{d \omega}{2 \pi i} \int \frac{d \mathbf{q}}{(2 \pi)^{2}} F_{V}^{*}(\omega, \mathbf{q}, L) F_{C}(\omega, \mathbf{q}, L), \\
& F_{C, V}(\omega, \mathbf{q}, L)=\int \frac{d k_{z}}{2 \pi} D(\omega) G_{C, V}\left(\omega, k_{z}, \mathbf{q}\right) \exp \left(i k_{z} L\right) .
\end{aligned}
$$

Both intra- and interband components of Eq. (14) can be calculated analytically for $\Omega_{C, V} \ll W_{C, V}$, where $\left(\Omega_{C}, \Omega_{V}\right)$, are distances between the sharp DOS peak of interfacial states and the top of the valence band or the bottom of the conduction band, respectively. In such a case the dominant contribution to the integrals in Eq. (14) is provided by the states with momenta $\mathbf{k}$, lying near the extreme points of the electron spectrum. At $\Omega_{C, V} \sim W_{C, V}$ only numerical calculation is possible, which needs integration over all momenta in the first Brillouin zone.

Here we consider only the case of the nondegenerated semiconductor in which the Fermi level lies inside the energy gap $E_{C}\left(K_{C}\right)>\varepsilon_{F}>E_{V}\left(K_{V}\right)$. Free carriers are absent in the spacer at zero temperature, so Eq. (14) describes only a contribution of the superexchange mechanism to IEC, but the contribution of the RKKY mechanism is absent. Explicit expressions for different components of integral $I(L)$ are presented in Appendix A.

Equation (14) describes the superexchange mechanism of IEC in frames of a simple two-band model. We see that the two different terms in $I(L)$ define the character of IEC. The 
intraband term $I_{1}(L)$ is always AFM, while the interband term $I_{2}(L) \cos \left(K_{0}^{z} L\right)$ is either FM, when $\mathbf{K}_{0}$ is strongly perpendicular to the growth axis, or oscillating with $L$ from FM to AFM. However, in a real spacer the situation is more complex, since the spatial group of crystalline symmetry of a semiconductor generates an irreducible star of vectors $\left\{\mathbf{K}_{C, V}\right\}$ with different orientations relative to the axis of growth. Consequently, a few equivalent maxima and minima exist in the electron spectrum. ${ }^{26}$ Due to the hybridization process with the transfer of large momenta at the interface, the different components of IEC (AFM, FM, and oscillating) emerge, from both the intra- and interband terms in $I(L)$. One can describe this in the following manner:

$$
\begin{aligned}
I(L)= & \sum_{C C^{\prime}}\left|\gamma_{C C^{\prime}}\right|^{2} A_{C}(L) \cos \left(K_{C C^{\prime}}^{z} L\right) \\
& +\sum_{V V^{\prime}}\left|\gamma_{V V^{\prime}}\right|^{2} A_{V}(L) \cos \left(K_{V V^{\prime}}^{z} L\right)+\sum_{C V}\left[\left|\gamma_{C V}\right|^{2} A_{C V}(L)\right. \\
& \left.+\left|\gamma_{V C}\right|^{2} A_{V C}(L)\right] \cos \left(K_{C V}^{z} L\right),
\end{aligned}
$$

where the sum is taken over the indices of band minima $\left(C, C^{\prime}\right)$ and maximums $\left(V, V^{\prime}\right)$, correspondingly, $A_{C}, A_{V}$, $A_{C V}$, and $A_{V C}$ are functions defined in Eq. (14). Two types of electron spectra, qualitatively corresponding to different situations in the real $\mathrm{Fe} / \mathrm{Si}$ systems, are considered in Appendix B.

\section{EFFECT OF INTERFACIAL AND COMPOSITIONAL DISORDER}

Up to here we considered only the systems with ideal interfaces and without compositional disorder inside the spacer. It is intuitively evident that the short-wave oscillatory term in $I(L)$ is extremely sensitive to interfacial roughness and composition fluctuations. Unfortunately, it is difficult to treat these factors directly in our microscopic model, since they destroy the in-plane translation invariance of the system and therefore the momentum $\mathbf{q}$ is no longer a good quantum number. However, using a simple phenomenological approach it is possible to qualitatively estimate modifications of IEC provided by the disorder.

A comparative analysis of the properties of interfaces in structures obtained with various technologies must take into account different scales of fluctuations of the surface relief at the Fe/Si boundaries. First, any method used for the growth leads to unavoidable short-scale fluctuations within several atomic layers near the $\mathrm{Fe} / \mathrm{Si}$ interface, caused by frustration of regular interatomic bonds (intermixing). This effect leads to the finite lifetime of interfacial states and may directly be included into the effective bandwidth $\left(W_{i}\right)$ and the effective DOS $\left[N_{i}(\varepsilon)\right]$. Second, any technology involves large-scale ("geometric") fluctuations of the surface relief (the so-called interfacial "roughness"). This roughness is usually described in terms of two statistical characteristics, the dispersion (vertical roughness) and the correlation length (lateral roughness) of fluctuations of the relief height. The value of vertical roughness is usually of order of some intermonolayer distances, while the lateral roughness can vary within three or- ders of magnitude: from several nanometers to several microns.

As was shown in Ref. 27 by using a phenomenological model of IEC for structures in which the spacer thickness variations are induced by the interfacial roughness, the oscillations of the "microscopic" exchange integral $I(L)$ with a short period $d_{s} \leqslant \sigma$, where $\sigma$ is a vertical roughness, produce both the bilinear $J_{1}(L)$ and biquadratic $J_{2}(L)$ components in $E_{e x}$ (see Sec. I). Obviously, in our model both these components of IEC formally result from phenomenological inclusion of an exchange stiffness of Fe layers in the exchange energy $\Delta \Omega_{e x}$ and averaging Eq. (11) over the spacer thickness variations. Unfortunately, such a procedure is correct only for structures with imperfect interfaces, where $\sigma$ $\geqslant 5-6 \AA$. For structures with almost ideal interfaces, where the vertical roughness is of order of one intermonolayer distance, the model of spacer thickness variation ${ }^{27}$ breaks down.

There are serious reasons for the system under investigation to be strongly inhomogeneous at micro- and macroscopic scales. A rather complicated "compositional" disorder may exist in the spacer, provided by breaking chemical bonds between silicon atoms, by formation of partially amorphous islands, or by spatial variations of the iron-silicide content in the spacer. Itinerant electrons move in the crystal potential composed of the periodic and disordered contributions; the latter has fluctuating components with different characteristic length scales. Even in systems without the iron diffusion, the short-range fluctuating potential of dangling bonds of silicon destroys perfect crystalline symmetry and periodicity. In systems with strong iron diffusion the longrange fluctuating potential of charged $\mathrm{Fe}$ ions induces additional disorder. Therefore there appear both longitudinal and transverse (parallel and perpendicular to the growth axis, respectively) variations of the band-structure parameters of the spacer.

Let us assume that within whatever method (for example, the coherent potential approximation) the fluctuations of a disorder potential at the microscopic (atomic) length scale are already included into the parameters of an electron spectrum. It can be shown that the DOS "tails" caused by disorder near the band edges only slightly modify the preexponent factors in the integrals $I_{1,2}(L)$. More severe modifications of IEC are produced by fluctuations of the momentum $\mathbf{K}_{0}$ in Eq. (14). At sufficiently large $L$ even small variation $\Delta K_{0}^{z}$ may provide the change in the sign of the oscillatory term in Eq. (14), if $\left|\Delta K_{0}^{z} L\right| \geqslant \pi$. Supposing for simplicity, that only isomorphic (i.e., without the local symmetry breaking) fluctuations are considered, we rewrite this condition in terms of relative variation $\Delta a / a$ of the intermonolayer distance $a: \frac{\Delta a}{a} \frac{L}{2 a} \geqslant 1$, which is satisfied at the spacer thickness $L \approx 10-20 \AA$ and $\Delta a / a \approx 0.2-0.3$.

Following the method of Ref. 27, after averaging Eq. (11) over the composition or thickness lateral fluctuations, we obtain the expression for $E_{e x}$ in the form

$$
\begin{gathered}
E_{e x}=\left\langle\Delta \Omega_{e x}\right\rangle=J_{1}(L) \mathbf{M}(-l) \mathbf{M}(+l)+J_{2}(L)[\mathbf{M}(-l) \mathbf{M}(+l)]^{2}, \\
J_{1}(L)=I_{1}(L), \quad J_{2}(L)=\lambda \xi\left[I_{2}(L)\right]^{2} / \Psi .
\end{gathered}
$$

Here $\Psi$ is the exchange stiffness of the Fe layer, $\xi$ is the lateral correlation length of composition or thickness fluctua- 
tions, and $\lambda$ is the numerical coefficient, which depends on details of averaging over either compositional or interfacial disorder.

\section{PHENOLOGICAL DESCRIPTION OF IEC}

Equation (16) describes the energy of IEC in terms of the "bilinear-biquadratic model" (see the Introduction), where coefficients $J_{1,2}(L)$ are complex functions of the spacer thickness. In principle, numerical calculations are needed to obtain these functions. However, already analytical estimations performed in Appendix A in the framework of a simplified two-band model for an electron spectrum can give some insight into the dependence of $J_{1,2}(L)$ on the spacer thickness. The intraband $A_{C}(L), A_{V}(L)$ and interband $A_{V C}(L), A_{C V}(L)$ integrals have opposite signs, different amplitudes, and characteristic lengths of decay at large distances. So, varying the band gap, effective masses of itinerant electrons in the bands and the position of spin-polarized interfacial states one can obtain complex $J_{1,2}(L)$ dependencies. Nevertheless, it is easy to demonstrate the peculiarities of IEC by using an even simpler phenomenological approach.

For example, one may qualitatively understand the origin of the maxima in the $J_{1}(L)$ dependence within the simplest exponential approximation. The rough approximation for the integral $J_{1}(L)=I_{1}(L)$ can be done within an exponential accuracy as $J_{1}(L)=A_{1} \exp \left(-L / L_{A}\right)-F_{1} \exp \left(-L / L_{F}\right)$, where $A_{1}$ and $F_{1}$ are positive pre-exponential factors, $L_{A, F}$ are characteristic lengths of AFM and FM components of superexchange coupling, respectively. We assume following experimental data that $L_{A}>L_{F}$, i.e., AFM type of IEC dominates at large $L$ and asymptotic behavior of $J_{1}(L)$ at $\left(L / L_{A}\right) \rightarrow \infty$ is $J_{1}(L) \approx \exp \left(-L / L_{A}\right)>0$.

If $\left(A_{1} / L_{A}\right)<\left(F_{1} / L_{F}\right)$, the $J_{1}(L)$ has the maximum at the length

$$
L_{1}^{*}=\frac{\ln \left(L_{A} / L_{F}\right)-\ln \left(A_{1} / F_{1}\right)}{L_{F}^{-1}-L_{A}^{-1}} .
$$

If $A_{1}-F_{1}>0$, then $J_{1}(L)>0$ and we have the AFM type of IEC for all $L$, as in Ref. 2 .

On the other hand, if $A_{1}-F_{1}<0$, then $J_{1}(L)$ passes through zero at

$$
L_{1}^{* *}=\frac{-\ln \left(A_{1} / F_{1}\right)}{L_{F}^{-1}-L_{A}^{-1}}<L_{1}^{*} .
$$

Note that $J_{1}(L)>0$ at $L>L_{1}^{* *}$, but $J_{1}(L)<0$ at $L<L_{1}^{* *}$ and the type of IEC changes from AFM to FM at $L=L_{1}^{* *}<L_{1}^{*}$ as $L$ decreases, as in Ref. 10.

Analogously, if one writes $I_{2}(L) \approx A_{2} \exp \left(-L / L_{A}\right)$ $-F_{2} \exp \left(-L / L_{F}\right)$, then the biquadratic exchange integral $J_{2}(L) \sim\left[I_{2}(L)\right]^{2}>0$ for all $L$. If $\left(A_{2} / L_{A}\right)<\left(F_{2} / L_{F}\right)$, then the maximum in $J_{2}(L)$ occurs at

$$
L_{2}^{*} \approx \frac{\ln \left(L_{A} / L_{F}\right)-\ln \left(A_{2} / F_{2}\right)}{L_{F}^{-1}-L_{A}^{-1}} .
$$

If $\ln \left(L_{A} / L_{F}\right) \gg\left\{\ln \left(A_{1} / F_{1}\right), \ln \left(A_{2} / F_{2}\right)\right\}$, the characteristic thicknesses $L_{1}^{*}$ and $L_{2}^{*}$ of the maxima of bilinear and biqua- dratic components of IEC are almost equal (see also Ref. 2).

Let us now discuss the IES results experimentally found in $\mathrm{Fe} / \mathrm{Fe}_{1-x} \mathrm{Si}_{x}$ structures ${ }^{2}$ in the framework of our model. The authors of Ref. 2 have shown that the IEC strength is greater than $5 \mathrm{~mJ} / \mathrm{m}^{2}$ for a nominally pure silicon spacer. This value of IEC is unusually high: one would expect it to be lower than in the case of metallic spacers where IEC is of $\sim 1 \mathrm{~mJ} / \mathrm{m}^{2}$. Bürgler et al. ${ }^{2}$ also exclude the formation of metallic iron silicide as the reason for the observed strong IEC and related this interesting result to the growth of a semiconductor spacer with high resistivity and apparent band gap. In the case of $x=1$ (silicon spacer) and sufficiently large spacer thickness $L$, when AFM coupling prevails, they found an exponential decrease of $J_{1}(L)$ with the decay length of $1.7 \AA .2,15$ For $0.5<x<1.0$ they observed a significant decrease of the strength of IEC by almost one order of magnitude and a shift of the IEC maximum to larger spacer thicknesses when nominal iron content $(1-x)$ increases from 0 to 0.5 . At the same time, the decay length of $J_{1}(L)$ increases and may reach the value $3.6 \AA$ at $x \approx 0.5$ (see Refs. 4 and 16). As reported in Ref. 10, IEC changes its sign at small spacer thicknesses. Unfortunately the details of the samples growing are not clearly exposed in Ref. 10, that impedes the interpretaion of significant differences between the results of Refs. 2 and 10 at the microscopic level.

Notice that direct comparison of our results with experimental data on IEC in $\mathrm{Fe} / \mathrm{Fe}_{1-x} \mathrm{Si}_{x}$ structures is impossible, since we do not have information on important microscopic characteristics of the system, in particular, the dependence of the energy gap $E_{g}$, effective masses $m_{C, V}$, and interfacial excitation energies $\Omega_{C, V}$ on the composition $x$. To qualitatively compare the measured IEC function $J_{1}(L)$ with the calculated one, we simplify theoretical expression for $J_{1}(L)$ by means of the above-mentioned "exponential" approximation, introducing some phenomenological parameters. The mean parameter influencing upon the behavior of $J_{1}(L)$ is the ratio $k$ $=L_{A} / L_{F}$, where $L_{A}$ and $L_{F}$ are characteristic lengths of AFM and FM components of IEC, respectively. As we demonstrated in Appendix A, these lengths are directly connected with microscopic parameters $\Omega_{C, V}, E_{g}$, and $m_{C, V}$.

Despite a lack of experimental data on this important characteristic of $\mathrm{Fe} / \mathrm{Fe}_{1-x} \mathrm{Si}_{x}$ structures, one can make some qualitative suppositions. First, the minimum of excitation energy $\Omega_{C, V}$ does not exceed the energy of interband process, which is of the order of the band-gap energy $E_{g}$; since $L_{A}$ $\sim\left[\Omega_{C, V}\right]^{-1 / 2}, L_{F} \sim\left[E_{g}\right]^{-1 / 2}$, and $k \sim\left[E_{g} / \Omega_{C, V}\right]^{-1 / 2}>1$, exponentially decaying AFM coupling dominates at large thickness $L$ in the structures $\mathrm{Fe} / \mathrm{Fe}_{1-x} \mathrm{Si}_{x}$ with a nonmetallic spacer $(0.5<x<1)$. Second, it would be natural to assume that in a system under consideration, upon increasing the iron content inside the spacer, the energy distance between the interfacial state and the conducting band bottom decreases due, for example, to the weakening of an interface potential and/or narrowing of the band gap. As a consequence, the characteristic length $L_{A}$ of the AFM component of IEC increases with Fe content; at least, such assumption is compatible with the experimental facts. The decrease of the IEC strength at small thicknesses, $L<L_{1}^{*}$, and the possible change of the IEC sign are likely related to the occurrence of 


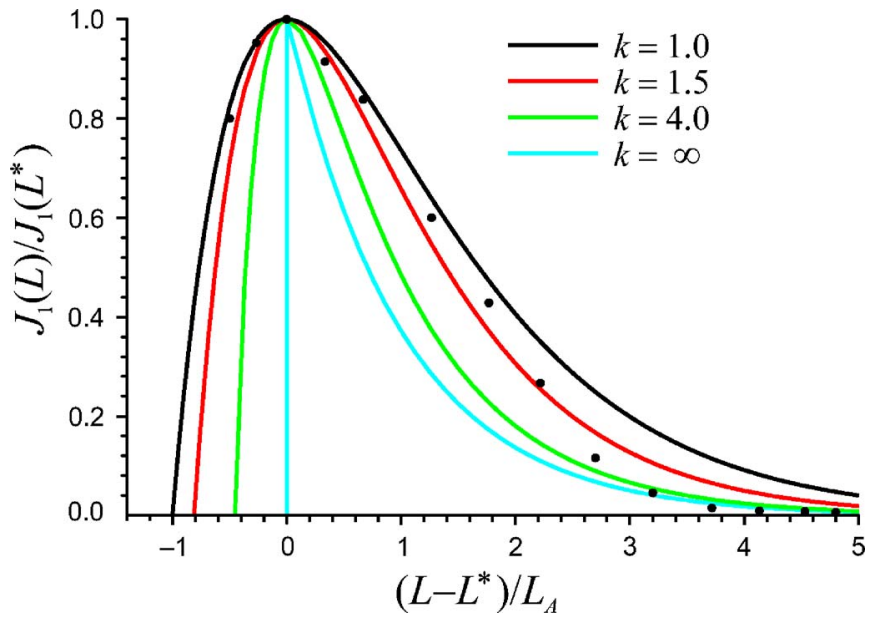

FIG. 1. (Color online) Behavior of the IEC energy as a function of the spacer thickness obtained in the "exponential" approximation. The $J_{1}(L)$ dependence is given in reduced units. The curves are presented for several values of the parameter $k=L_{A} / L_{F}$. The circles show the experimental $J_{1}(L)$ data from Ref. 2 for structures with a nominally pure silicon spacer normalized to the maximum magnitude attained at $L=L^{*}=7.7 \AA$. $^{2}$ The spacer thickness is given in terms of dimensionless variable $L / L_{A}$, where $L_{A}$ is chosen to be equal to $3.0 \AA$.

the FM component of IEC, fully or partially connected with the intraband contribution to superexchange. It should be also mentioned that there exist other mechanisms of indirect FM coupling for real systems, coming from pinholes and spontaneous or induced magnetic ordering in the spacer, the influence of which has to grow when the Fe content in the spacer is increased and its thickness is reduced. ${ }^{2}$ Thus three observations $s^{2,10,15}$ - the increase of the maximum coupling strength with $x$, the decrease of $L_{1}^{*}$ with $x$, and the alteration of the IEC type (from AFM to FM) — can be qualitatively described on the basis of the "exponential" approximation.

Theoretical dependence of $J_{1}$ on $L$ for different $k$ $=L_{A} / L_{F}$ is displayed in Fig. 1 ; one can see that AFM coupling is exponentially decaying at large $L$, attains the maximum at intermediate $L$ and is limited by dominant FM coupling at small $L$. Thoroughly measured dependence of $J_{1}$ vs $L$ for $\mathrm{Fe} / \mathrm{Si} / \mathrm{Fe}$ trilayer with nominally pure silicon spacer $^{2}$ is also plotted in Fig. 1. In the region of intermediary thicknesses, $6-8<L<14-15 \AA$ the experimental points are fitted with the "exponentially" approximated $J_{1}$ in the best manner if the characteristic length $L_{A}$ is taken to be equal to $\approx 3.0 \AA$ and the ratio $k=L_{A} / L_{F}$ lies in the interval from 1 to 1.5 , i.e., the characteristic length $L_{F}$ varies from $\approx 2.0 \AA$ to $\approx 3.0 \AA$. However, in the region of large thicknesses, $L>14-15 \AA$, the simple exponential approximation with $L_{A}=1.8 \AA$ is rather suitable.

This fact could be explained by a loss of homogeneity of the spacer material, which is due, for example, to inevitable interdiffusion at stage of growing. Of course, the epitaxial growth obtained by co-evaporation allows one to produce the $\mathrm{Fe} / \mathrm{Fe}_{1-x} \mathrm{Si}_{x} / \mathrm{Fe}$ samples with a high degree of structural and compositional homogeneity; at the same time, X-ray spectroscopy demonstrates both the semiconductor nature and presence of iron silicides even for nominally pure $\mathrm{Si}$ interlayer. $^{2}$ A relatively small concentration of iron in the interfacial region would be enough to enhance the FM component of IEC, produce significant shift of the band edges, diminish the minimum of $\Omega_{C, V}$, and increase the length $L_{A}$ at small $L$.

Obviously, the decrease of $\Omega_{C, V}$ and the corresponding increase of $L_{A}$ in systems with small spacer thicknesses may be also provided by perturbation of an electron spectrum near the interface, due to the proximity effect from the interfacial potential and/or the lattice distortion, which takes place even in homogeneous materials.

\section{CONCLUSION}

In this work we have proposed the superexchange mechanism of interlayer exchange coupling (IEC) in Fe/Si structures, based on the following three principal ideas.

(i) Contact induced ferromagnetic (FM) phase of c-FeSi and spin-polarized interfacial states are formed at the $\mathrm{Fe} / \mathrm{Si}$ boundaries.

(ii) Exchange coupling between Fe layers is effectuated by means of the superexchange of spin-polarized interfacial states through the nonmagnetic semiconductor spacer.

(iii) The complex character of IEC dependence on the spacer thickness and composition is due to the competition between antiferromagnetic (AFM) and FM components of superexchange.

Our model qualitatively explains most of the existing experimental results, however, the analytical calculations have been carried out only at $T=0$ in the framework of a simple two-band scheme of electron spectrum. The studies of IEC at $T \neq 0$ and more realistic electron spectrum are highly desirable. Despite the qualitative description of existing experimental data we would like to note that our approach is not well suited for spacers, strongly enriched with iron and having metallic properties, for example, when the metallic compound $\mathrm{Fe}_{1-x} \mathrm{Si}_{x}$ with $x \geqslant 0.5$ (see Ref. 13) is formed inside the spacer due to strong diffusion of Fe. First, the role of "metallic" bands significantly rises in systems with metallic spacers, where the RKKY mechanism of the coupling remains essential, while the superexchange provides only small AFM "bias" of the IEC integral. ${ }^{23}$ Second, it is well known that the features of electron spectrum (in particular, the empty narrow band, forming a sharp DOS peak near the Fermi level) may strongly enhance paramagnetic spin fluctuations in the iron-rich silicides, ${ }^{28}$ thus making the role of spin fluctuations in the IEC coupling very important. ${ }^{29}$ Third, the "loose spins" mechanism ${ }^{30}$ plays a significant role in the IEC across metallic spacers, as has been demonstrated in Ref. 16.

We apply our model to estimate the IEC dependence on the spacer thickness for $\mathrm{Fe} / \mathrm{Si}$ and $\mathrm{Fe} / \mathrm{Fe}_{1-x} \mathrm{Si}_{x}$ (with 0.5 $<x<1$ ) layered structures. Our approach is formally valid only for systems with a sufficiently large energy gap $E_{g}$ of semiconductor spacer, $E_{g} \gg W_{i}$, where $W_{i}$ is the bandwidth of interfacial states. Strictly speaking, this condition may be realized only for contents $x>x_{\text {crit }}$, where $x_{\text {crit }} \geqslant 0.5$ is the critical content corresponding to the semiconductorsemimetal transition in $\mathrm{Fe}_{1-x} \mathrm{Si}_{x}$. 
In contrast to the $\mathrm{Fe} / \mathrm{Si}$ systems, $\mathrm{Co} / \mathrm{Si}$ nanostructures have not been studied in detail. The obtained experimental results are contradictory and show no clear evidence of IEC. This can be owing to strong diffusion processes which are difficult to be controlled and to the formation of the cobaltsilicide (possibly, c-CoSi) near the Co/Si interfaces. ${ }^{31-34} \mathrm{On}$ the other hand, evident AFM coupling has been recently observed in $\mathrm{Co}_{1-x} \mathrm{Si}_{x} / \mathrm{Si}$ multilayers with $x \approx 0.26 .{ }^{35}$ Both these facts may be qualitatively explained in our model, if we take into consideration the band structure of c-CoSi. This monosilicide is isomorphic to c-FeSi, however, has one additional valence electron and shows the DOS peak below the Fermi level (in c-FeSi this peak is located above the Fermi level). As a result, strong charge redistribution near $\mathrm{Co} / \mathrm{Si}$ interface, which shifts down the DOS peak, cannot result in spinpolarized interfacial states as well as in contact induced phase FM c-CoSi. So, the above discussed mechanism of IEC is not valid. However, in metallic $\mathrm{Co}_{1-x} \mathrm{Si}_{x}$ the Fermi level strongly decreases with increasing $x$; thus the conditions of appearance of spin-polarized states near the $\mathrm{Co}_{1-x} \mathrm{Si}_{x} / \mathrm{Si}$ interfaces become satisfied, "switching" the superexchange mechanism of IEC at $x \approx 0.26$. $^{35}$

\section{ACKNOWLEDGMENTS}

We are grateful to P.M. Echenique for valuable discussions. The work was partially supported by the UPV/EHU, the Departamento de Educación del Gobierno Vasco, and MCyT (Grant No. FIS 2004-06490-C03-01).

\section{APPENDIX A}

Let us approximate the narrow peak in the DOS, $N(\varepsilon)$, of interfacial states by the local level with energy $\varepsilon_{0}$ and write $N_{i}(\varepsilon) \cong n_{i} \delta\left(\varepsilon-\varepsilon_{0}\right)$, where $n_{i}$ is the number of states in the peak. Such approximation seems to be reasonable, if the peak width $W_{i} \ll\left(W_{C, V}, J_{i} M\right)$. The interfacial exchange potential $J_{i} M$ (below we define $J_{i} M>0$ ) splits the level $\varepsilon_{0}$ in two sublevels. We define the position of the Fermi level as $\varepsilon_{F}=0$, so the energies of filled ("spin-up") and empty ("spindown") sublevels are $\varepsilon_{0}-J_{i} M<0$ and $\varepsilon_{0}+J_{i} M>0$, respectively.

Let us calculate the intraband components of IEC. In the expressions for $A_{C}(L)$ or $A_{V}(L)$ (see Sec. IV) one may at first carry out integration over complex frequency $\omega$ along the contour closed in upper half plane and then integration over transverse momentum $k_{z}$ from $-\infty$ to $+\infty$. For the superexchange integral $A_{C}(L)$ one obtains the following expression:

$$
\begin{aligned}
A_{C}(L)= & \frac{n_{i}^{2} m_{C}}{16 M^{2}} \int \frac{d \mathbf{q}}{(2 \pi)^{2}}\left(\frac{1}{J_{i} M \Omega_{C}(\mathbf{q})}+\frac{L \sqrt{2 m_{C}}}{\left[\Omega_{C}(\mathbf{q})\right]^{3 / 2}}\right) \\
& \times \exp \left[-2 L \sqrt{2 m_{C} \Omega_{C}(\mathbf{q})}\right],
\end{aligned}
$$

where $\Omega_{C}(\mathbf{q})=\Omega_{C}+\frac{q^{2}}{2 m_{C}}, \Omega_{C}=E_{C}\left(\mathbf{K}_{C}\right)-\varepsilon_{0}+J_{i} M$. The remaining integral in Eq. (A1) over the momentum q, lying into an interface plane, can be expressed in a fairly compact way:

$$
A_{C}(L)=\frac{n_{i}^{2} m_{C}^{2}}{8 \pi M^{2}}\left[\frac{\exp \left(-L / L_{C}\right)}{2 \Omega_{C}}-\frac{\operatorname{Ei}\left(-L / L_{C}\right)}{J_{i} M}\right],
$$

where $L_{C}=\left(2 \sqrt{2 m_{C} \Omega_{C}}\right)^{-1}, \operatorname{Ei}(t)$ is the integral exponent function:

$$
\operatorname{Ei}(t)=-\int_{-\infty}^{t} \frac{e^{x}}{x} d x
$$

The contribution $\sim A_{C}(L)$ is due to the indirect exchange interaction of FM layers across virtual excitations of two electrons with opposite spin projections (one electron from each interface) from interfacial states to the conduction band of the spacer.

Analogously, one obtains an expression for the superexchange integral $A_{V}(L)$,

$$
A_{V}(L)=\frac{n_{i}^{2} m_{V}^{2}}{8 \pi M^{2}}\left[\frac{\exp \left(-L / L_{V}\right)}{2 \Omega_{V}}-\frac{\operatorname{Ei}\left(-L / L_{V}\right)}{J_{i} M}\right],
$$

where $\Omega_{V}=\left|E_{V}\left(\mathbf{K}_{V}\right)\right|+\varepsilon_{0}+J_{i} M$ and $L_{V}=\left(2 \sqrt{2 m_{V} \Omega_{V}}\right)^{-1}$.

The contribution $\sim A_{V}(L)$ is due to the indirect exchange interaction of FM layers across virtual excitations of two holes with opposite spin projections (one hole from each interface) from interfacial states to the valence band of the spacer.

The asymptotic behavior of functions $A_{C, V}(L)$ at large distances $L / L_{C, V}$ has the form

$$
A_{C, V}(L)=\frac{n_{i}^{2} m_{C, V}^{2}}{8 \pi M^{2}}\left[\frac{1}{2 \Omega_{C, V}}+\frac{L_{C, V}}{J_{i} M L}\right] e^{-L / L_{C, V}} .
$$

The values $\Omega_{C, V}$ can be considered as excitation energies of quasiparticles in the processes of their tunneling from "donor" and "acceptor" interfacial states to the conduction and valence band of the spacer.

We see that intraband contribution $A_{C, V}(L)$ in IEC is always AFM and the phenomenological length $L_{A}$ in Sec. VI may be associated with the maximal length $L_{C}$ or $L_{V}$ in Eqs. (A2) and (A3). Note that $\left\{L_{C, V} \sim a \sqrt{W_{C, V} / \Omega_{C, V}}\right\} \gg a$, where $a$ is the lattice parameter. In other words, due to the hybridization with the states of the spacer, the interfacial states become less localized in the $z$ direction, with the wave functions extended over a finite range, comparable to the spacer thickness. The overlap of the "tails" of such wave functions leads to the long-range exchange interaction $I_{1}(L)$, when the free carriers in the spacer are yet absent.

Calculation of the interband contribution, unlike the intraband one, is appeared to be more complicated. After integration over complex frequency $\omega$, one obtains the following expression for the interband contribution $A_{C V}(L)$ : 


$$
\begin{aligned}
A_{C V}(L)= & -\frac{n_{i}^{2}}{8 M^{2}} \int \frac{d \mathbf{q}}{(2 \pi)^{2}} \int \frac{d k_{z}}{2 \pi} \int \frac{d k_{z}^{\prime}}{2 \pi} \frac{\exp \left[i\left(k_{z}-k_{z}^{\prime}\right) L\right]}{k_{z}^{2} / 2 m_{C}+k_{z}^{\prime 2} / 2 m_{V}+E_{g}(\mathbf{q})} \\
& \times\left\{\frac{1}{J_{i} M\left[\Omega_{C}(\mathbf{q})+k_{z}^{2} / 2 m_{C}\right]}+\frac{1}{\left[\Omega_{C}(\mathbf{q})+k_{z}^{2} / 2 m_{C}\right]^{2}}+\frac{1}{J_{i} M\left[\Omega_{V}(\mathbf{q})+k_{z}^{\prime 2} / 2 m_{V}\right]}+\frac{1}{\left[\Omega_{V}(\mathbf{q})+k_{z}^{\prime 2} / 2 m_{V}\right]^{2}}\right\},
\end{aligned}
$$

where notations $E_{g}(\mathbf{q})=E_{g}+\left(\frac{1}{m_{C}}+\frac{1}{m_{V}}\right) \frac{q^{2}}{2}, \Omega_{C}(\mathbf{q})=\Omega_{C}+\frac{q^{2}}{2 m_{C}}, \Omega_{V}(\mathbf{q})=\Omega_{V}+\frac{q^{2}}{2 m_{V}}$ are introduced. After transformations $k_{z}-k_{z}^{\prime}=k$ and $\sqrt{\frac{m_{V}}{m_{C}}} k_{z}+\sqrt{\frac{m_{C}}{m_{V}}} k_{z}^{\prime}=K$ the integral (A5) is rewritten in the form

$$
\begin{aligned}
A_{C V}(L)= & -\frac{n_{i}^{2}}{8 M^{2}} \frac{\left(m_{C}+m_{V}\right)^{4}}{\sqrt{m_{C} m_{V}}} \int \frac{d \mathbf{q}}{(2 \pi)^{2}} \int \frac{d k}{2 \pi} \int \frac{d K}{2 \pi} \frac{e^{i k L}}{k^{2}+K^{2}+2\left(m_{C}+m_{V}\right) E_{g}(\mathbf{q})} \\
& \times\left\{\frac{1}{J_{i} M\left[\left(K-\sqrt{m_{C} / m_{V}} k\right)^{2}+2\left(m_{C}+m_{V}\right)^{2} / m_{V} \Omega_{C}(\mathbf{q})\right]}+\frac{2\left(m_{C}+m_{V}\right)^{2} / m_{V}}{\left[\left(K-\sqrt{m_{C} / m_{V} k}\right)^{2}+2\left(m_{C}+m_{V}\right)^{2} / m_{V} \Omega_{C}(\mathbf{q})\right]^{2}}\right. \\
& \left.+\frac{1}{J_{i} M\left[\left(K+\sqrt{m_{V} / m_{C}} k\right)^{2}+2\left(m_{C}+m_{V}\right)^{2} / m_{C} \Omega_{V}(\mathbf{q})\right]}+\frac{2\left(m_{C}+m_{V}\right)^{2} / m_{C}}{\left[\left(K+\sqrt{\left.\left.m_{V} / m_{C} k\right)^{2}+2\left(m_{C}+m_{V}\right)^{2} / m_{C} \Omega_{V}(\mathbf{q})\right]^{2}}\right.\right.}\right\} .
\end{aligned}
$$

The integral (A6) is cumbersome and it is impossible to find its explicit analytical expression, except for some limiting cases. Carrying out the integration over variable $K$ we see that the function of variable $k$ has the poles on the imaginary axis in the upper half plane at points

$$
k_{C}^{ \pm}=i \sqrt{2} \mid \sqrt{m_{V}\left[E_{g}(\mathbf{q})-\Omega_{C}(\mathbf{q})\right]} \pm \sqrt{m_{C} \Omega_{C}(\mathbf{q})}
$$

for the first and second terms and at points

$$
k_{V}^{ \pm}=i \sqrt{2} \mid \sqrt{m_{C}\left[E_{g}(\mathbf{q})-\Omega_{V}(\mathbf{q})\right]} \pm \sqrt{m_{V} \Omega_{V}(\mathbf{q})}
$$

for the third and fourth terms, respectively. It can be shown that the contributions given by poles $k_{C, V}^{-}$are exactly canceled. On the other hand, the pole nearest to the real axis dominates in the leading asymptotic estimation of the integral (A6) at large $L \gg L_{C, V}$, and we have

$$
\begin{aligned}
A_{C V}(L)= & -\frac{n_{i}^{2}}{M^{2}} \int \frac{d \mathbf{q}}{(2 \pi)^{2}}\left\{\Lambda_{C}(\mathbf{q}) \exp [\right. \\
& \left.-\sqrt{2}\left(\sqrt{m_{V}\left[E_{g}(\mathbf{q})-\Omega_{C}(\mathbf{q})\right]}+\sqrt{m_{C} \Omega_{C}(\mathbf{q})}\right) L\right] \\
& +\Lambda_{V}(\mathbf{q}) \exp \left[-\sqrt{2}\left(\sqrt{m_{C}\left[E_{g}(\mathbf{q})-\Omega_{V}(\mathbf{q})\right]}\right.\right. \\
& \left.\left.\left.+\sqrt{m_{V} \Omega_{V}(\mathbf{q})}\right) L\right]\right\},
\end{aligned}
$$

where coefficients $\Lambda_{C, V}\left(m_{C}, m_{V}, \mathbf{q}\right)$ are complicated functions of $\left(m_{C}, m_{V}, \mathbf{q}\right)$ not depending on $L$. To simplify calculations, we assume that $m_{C}=m_{V}=m$ and arrive in the leading exponential approximation to the expression

$$
\begin{aligned}
A_{C V}(L)= & -\frac{n_{i}^{2} m^{2}}{8 \pi E_{g} M^{2}} \times\left\{\left[\frac{\sqrt{\Omega_{C}} \sqrt{\Omega_{V}-2 J_{i} M}}{J_{i} M L}+\frac{\sqrt{2 m}}{2} \mid \sqrt{\Omega_{C}}\right.\right. \\
& \left.-\sqrt{\Omega_{V}-2 J_{i} M}\right] L_{1}^{\text {inter }} \exp \left(-\frac{L}{L_{1}^{\text {inter }}}\right) \\
& +\left[\frac{\sqrt{\Omega_{V}} \sqrt{\Omega_{C}-2 J_{i} M}}{J_{i} M L}+\frac{\sqrt{2 m}}{2} \mid \sqrt{\Omega_{V}}\right.
\end{aligned}
$$

$$
\left.\left.-\sqrt{\Omega_{C}-2 J_{i} M}\right] L_{2}^{\text {inter }} \exp \left(-\frac{L}{L_{2}^{\text {inter }}}\right)\right\},
$$

where

$$
\begin{aligned}
& {\left[L_{1}^{\text {inter }}\right]^{-1}=\sqrt{2 m}\left(\sqrt{\Omega_{C}}+\sqrt{\Omega_{V}-2 J_{i} M}\right),} \\
& {\left[L_{2}^{\text {inter }}\right]^{-1}=\sqrt{2 m}\left(\sqrt{\Omega_{V}}+\sqrt{\Omega_{C}-2 J_{i} M}\right) .}
\end{aligned}
$$

It can be shown that at different effective masses of electron and holes $\left[L_{1}^{\text {inter }}\right]^{-1}=\sqrt{2 m_{C} \Omega_{C}}+\sqrt{2 m_{V}\left(\Omega_{V}-2 J_{i} M\right)}$ and $\left[L_{2}^{\text {inter }}\right]^{-1}=\sqrt{2 m_{V} \Omega_{V}}+\sqrt{2 m_{C}\left(\Omega_{C}-2 J_{i} M\right)}$, respectively.

The contribution $\sim A_{C V}$ is due to indirect exchange interaction of FM layers across electron-hole virtual excitations, in which, for example, the electron with spin "up" and the hole with spin "down" are emitted from the "left" interfacial states into the conduction and valence band of the spacer, respectively, in order to be absorbed by the "right" interfacial states. Obviously, analogous process with opposites spins of electron and hole take place in the system, as well as processes of electron-hole virtual excitations in the opposite direction, i.e., from the "right" to the "left" interfacial states.

We see that the intraband contribution $A_{C V}(L)$ in IEC is always FM and the phenomenological length $L_{F}$ in Sec. VI may be associated with the maximal length in Eq. (A10), $L_{1}^{\text {inter }}$ or $L_{2}^{\text {inter }}$ (note the lengths $L_{1,2}^{\text {inter }} \gg a$ ).

The absolute values of intra- and interband contributions in IEC, as well as their characteristic lengths are of the same order and strongly depend on details of an electron spectrum of the spacer (effective masses, energy gap) and positions of spin-polarized interfacial states $\left(\varepsilon_{0} \pm J_{i} M\right)$. Therefore the sign of IEC results from the competition between the AFM and FM components.

In our calculations we formally used the method proposed in Ref. 36 to describe ferromagnetism from localized magnetic impurities in the two-band model developed for semiconductors. However, our system has important differences from that studied in Ref. 36. First, we have considered 
weakly correlated interfacial states with large spin splitting $\left(J_{i} M \gg U\right)$ induced by the exchange field of adjacent FM layer. These states have the structure very different from that in Ref. 36, where the mechanism of indirect exchange between the Anderson's magnetic impurities with very strong one-site electron repulsion $(U \rightarrow \infty)$ was discussed. Second, in the impurity situation the potential of hybridization is not a periodic function in the $(x, y)$ plane, and integration over transverse momentum is carry out in a different way.

\section{APPENDIX B}

Let us analyze the structure of the IEC integral for systems with complex electron spectrum, taking into account effects of crystalline symmetry.

\section{Model I}

Let us have the electron spectrum with the valence-band maximum at the $\Gamma$ point and the conduction-band minima, lying near the $X$ points of the first Brillouin zone of the diamond lattice. The irreducible star $\left\{\mathbf{K}_{C}\right\}$ contains the vectors

$$
\begin{aligned}
& \mathbf{K}_{ \pm 1}=\frac{\pi}{2 a}(1 \pm \delta, 0,0), \\
& \mathbf{K}_{ \pm 2}=\frac{\pi}{2 a}(0,1 \pm \delta, 0), \\
& \mathbf{K}_{ \pm 3}=\frac{\pi}{2 a}(0,0,1 \pm \delta),
\end{aligned}
$$

where $\delta \ll 1, a$ is the intermonolayer distance; $\mathbf{K}_{V}=0 .{ }^{26} \mathrm{In}$ this case Eq. (15) can be written in the form

$$
\begin{gathered}
I(L)=I_{1}(L)+I_{2}(L) \cos \left(K_{0}^{z} L\right), \quad K_{0}^{z}=\frac{\pi}{2 a}, \\
I_{1}(L)=I_{1}^{\prime}(L)+I_{1}^{\prime \prime}(L) \cos \left(\frac{\pi}{a} \delta L\right) \\
I_{2}(L)=I_{2}^{\prime}(L) \cos \left(\frac{\pi}{2 a} \delta L\right) \\
I_{1}^{\prime}(L)=\left|\gamma_{V V}\right|^{2} A_{V}(L)+\left[6\left|\gamma_{C C}\right|^{2}+2\left|\gamma_{C C}^{\prime \prime}\right|^{2}\right] A_{C}(L) \\
+\left|\gamma_{C V}^{\prime}\right|^{2} A_{C V}(L)+\left|\gamma_{V C}^{\prime}\right|^{2} A_{V C}(L), \\
I_{1}^{\prime \prime}(L)=2\left|\gamma_{C C}\right|^{2} A_{C}(L), \\
I_{2}^{\prime}(L)=4\left|\gamma_{C C}^{\prime}\right|^{2} A_{C}(L)+2\left|\gamma_{C V}\right|^{2} A_{C V}(L)+2\left|\gamma_{V C}\right|^{2} A_{V C}(L),
\end{gathered}
$$

where

$$
\begin{aligned}
& \left|\gamma_{V V}\right|^{2}=\left|\gamma_{V V}(0)\right|^{2}, \\
& \left|\gamma_{C C}\right|^{2}=\left|\gamma_{C C}(0)\right|^{2} \\
& \left|\gamma_{V C}\right|^{2}=\left|\gamma_{V C}(0)\right|^{2}, \\
& \left|\gamma_{C C}^{\prime}\right|^{2}=\left|\gamma_{C C}\left(\mathbf{K}_{+1}\right)\right|^{2}+\left|\gamma_{C C}\left(\mathbf{K}_{+2}\right)\right|^{2}+\left|\gamma_{C C}\left(\mathbf{K}_{-1}\right)\right|^{2} \\
& +\left|\gamma_{C C}\left(\mathbf{K}_{-2}\right)\right|^{2} \text {, } \\
& \left|\gamma_{C V}^{\prime}\right|^{2}=\left|\gamma_{C V}\left(\mathbf{K}_{+1}\right)\right|^{2}+\left|\gamma_{C V}\left(\mathbf{K}_{+2}\right)\right|^{2}+\left|\gamma_{C V}\left(\mathbf{K}_{-1}\right)\right|^{2} \\
& +\left|\gamma_{C V}\left(\mathbf{K}_{-2}\right)\right|^{2} \text {, } \\
& \left|\gamma_{V C}^{\prime}\right|^{2}=\left|\gamma_{V C}\left(\mathbf{K}_{+1}\right)\right|^{2}+\left|\gamma_{V C}\left(\mathbf{K}_{+2}\right)\right|^{2}+\left|\gamma_{V C}\left(\mathbf{K}_{-1}\right)\right|^{2} \\
& +\left|\gamma_{V C}\left(\mathbf{K}_{-2}\right)\right|^{2} \text {, } \\
& \left|\gamma_{C C}^{\prime \prime}\right|^{2}=\left|\gamma_{C C}\left(\mathbf{K}_{+1}+\mathbf{K}_{+2}\right)\right|^{2}+\left|\gamma_{C C}\left(\mathbf{K}_{-1}+\mathbf{K}_{+2}\right)\right|^{2}+\mid \gamma_{C C}\left(\mathbf{K}_{+1}\right. \\
& \left.+\mathbf{K}_{-2}\right)\left.\right|^{2}+\left|\gamma_{C C}\left(\mathbf{K}_{-1}+\mathbf{K}_{-2}\right)\right|^{2} \text {. }
\end{aligned}
$$

\section{Model II}

The spectrum has the valence-band maxima at the $X$ point and the conduction-band minima at the $M$ point of the first Brillouin zone of the body-centered-cubic lattice. The irreducible stars are composed of vectors $\left\{\mathbf{K}_{V}\right\}$ :

$$
\begin{aligned}
& \mathbf{K}_{1 V}=\frac{\pi}{b}(1,0,0), \\
& \mathbf{K}_{2 V}=\frac{\pi}{b}(0,1,0), \\
& \mathbf{K}_{3 V}=\frac{\pi}{b}(0,0,1),
\end{aligned}
$$

and $\left\{\mathbf{K}_{C}\right\}$ : 


$$
\begin{aligned}
& \mathbf{K}_{1 C}=\frac{\pi}{b}(1,1,0), \\
& \mathbf{K}_{2 C}=\frac{\pi}{b}(0,1,1), \\
& \mathbf{K}_{3 C}=\frac{\pi}{b}(1,0,1),
\end{aligned}
$$

where $b$ is the bcc lattice parameter. ${ }^{26}$ We can write Eq. (15) in the form

$$
\begin{gathered}
I(L)=I_{1}(L)+I_{2}(L) \cos \left(K_{0}^{z} L\right), K_{0}^{z}=\frac{\pi}{2 b}, \\
I_{1}(L)=\left[3\left|\gamma_{V V}\right|^{2}+2\left|\gamma_{V V}^{\prime \prime}\right|^{2}\right] A_{V}(L)+\left[3\left|\gamma_{C C}\right|^{2}+2\left|\gamma_{C C}^{\prime \prime}\right|^{2}\right] A_{C}(L) \\
+2\left|\gamma_{V C}\right|^{2} A_{V C}(L)+2\left|\gamma_{V C}^{\prime}\right|^{2} A_{V C}(L), \\
I_{2}(L)=2\left|\gamma_{V V}^{\prime}\right|^{2} A_{V}(L)+2\left|\gamma_{C C}^{\prime}\right|^{2} A_{C}(L)+\left[3\left|\gamma_{V C}^{\prime \prime}\right|^{2}\right. \\
\left.+2\left|\gamma_{V C}\right|^{2}\right] A_{V C}(L)+\left[3\left|\gamma_{C V}^{\prime \prime}\right|^{2}+2\left|\gamma_{C V}\right|^{2}\right] A_{C V}(L),
\end{gathered}
$$

where

$$
\begin{gathered}
\left|\gamma_{V V}\right|^{2}=\left|\gamma_{V V}(0)\right|^{2}, \\
\left|\gamma_{C C}\right|^{2}=\left|\gamma_{C C}(0)\right|^{2}, \\
\left|\gamma_{V C}\right|^{2}=\left|\gamma_{V C}(0)\right|^{2}, \\
\left|\gamma_{C C}^{\prime}\right|^{2}=\left|\gamma_{C C}\left(\mathbf{K}_{1}\right)\right|^{2}+\left|\gamma_{C C}\left(\mathbf{K}_{2}\right)\right|^{2}, \\
\left|\gamma_{V V}^{\prime}\right|^{2}=\left|\gamma_{V V}\left(\mathbf{K}_{1}\right)\right|^{2}+\left|\gamma_{V V}\left(\mathbf{K}_{2}\right)\right|^{2},
\end{gathered}
$$

$$
\begin{gathered}
\left|\gamma_{C V}^{\prime}\right|^{2}=\left|\gamma_{C V}\left(\mathbf{K}_{1}\right)\right|^{2}+\left|\gamma_{C V}\left(\mathbf{K}_{2}\right)\right|^{2} \\
\left|\gamma_{V C}^{\prime}\right|^{2}=\left|\gamma_{V C}\left(\mathbf{K}_{1}\right)\right|^{2}+\left|\gamma_{V C}\left(\mathbf{K}_{2}\right)\right|^{2} \\
\left|\gamma_{C C}^{\prime \prime}\right|^{2}=\left|\gamma_{C C}\left(\mathbf{K}_{1}+\mathbf{K}_{2}\right)\right|^{2}, \\
\left|\gamma_{V V}^{\prime \prime}\right|^{2}=\left|\gamma_{V V}\left(\mathbf{K}_{1}+\mathbf{K}_{2}\right)\right|^{2}, \\
\left|\gamma_{V C}^{\prime \prime}\right|^{2}=\left|\gamma_{V C}\left(\mathbf{K}_{1}+\mathbf{K}_{2}\right)\right|^{2}, \\
\left|\gamma_{C V}^{\prime \prime}\right|^{2}=\left|\gamma_{C V}\left(\mathbf{K}_{1}+\mathbf{K}_{2}\right)\right|^{2}
\end{gathered}
$$

This model can be qualitatively suitable to analyze IEC in structures with strong diffusion of iron, when PM c-FeSi is extended over all the spacer thickness. Since $b \approx 2.8-2.9 \AA,^{20}$ the period of short-wave oscillations of $I(L)$ can be estimated as $d_{s} \approx 5.6-5.8 \AA$. Obviously, in model II we neglect peculiarities of the real electron spectrum of PM c-FeSi. Strictly speaking, the edges of "conduction" and "valence" bands near the $M$ and $X$ points are separated not by a true gap, but a "pseudogap" $E_{g} \sim 0.5 \mathrm{eV}$, the Fermi level is situated on $\sim 0.05 \mathrm{eV}$ below the bottom of conduction band $E_{C}\left(K_{C}\right)$ at the $M$ point. There are also other bands, which form the Fermi surface and which are responsible for the metallic character of conductivity. ${ }^{20}$ We presume that the role of such "metallic" bands in the superexchange mechanism of IEC is negligible, due to their low DOS. We do not discuss here the effect of the upper conduction band that has a very high effective mass and lies at $\sim 0.3 \mathrm{eV}$ above the minimum of the lowest conduction band at the $M$ point. It can be shown ${ }^{37}$ that the role of the upper conduction band becomes significant only for $L<5 \AA$.

We hope that both these models considered qualitatively describe the superexchange through the spacers with different spatial symmetry and grown in different conditions.
${ }^{1}$ I. Žutić, J. Fabian, and S. Das Sarma, Rev. Mod. Phys. 76, 323 (2004).

${ }^{2}$ D. E. Bürgler, M. Buchmeier, S. Cramm, S. Eisebitt, R. R. Gareev, P. Grünberg, C. L. Jia, L. L. Pohlmann, R. Schreiber, M. Siegel, Y. L. Qin, and A. Zimina, J. Phys.: Condens. Matter 15, S443 (2003).

${ }^{3}$ B. Briner and M. Landolt, Phys. Rev. Lett. 73, 340 (1994).

${ }^{4}$ J. J. de Vries, J. Kohlhepp, F. J. A. den Broeder, R. Coehoorn, R. Jungblut, A. Reinders, and W. J. M. de Jonge, Phys. Rev. Lett. 78, 3023 (1997)

${ }^{5}$ G. J. Strijkers, J. T. Kohlhepp, H. J. M. Swagten, and W. J. M. de Jonge, Phys. Rev. Lett. 84, 1812 (2000).

${ }^{6}$ E. E. Fullerton and S. D. Bader, Phys. Rev. B 53, 5112 (1996).

${ }^{7}$ J. Kohlhepp, M. Valkier, A. van der Graaf, and F. J. A. den Broeder, Phys. Rev. B 55, R696 (1997).

${ }^{8}$ R. W. E. van de Kruijs, M. Th. Rekveldt, H. Fredrikze, J. T. Kohlhepp, J. K. Ha, and W. J. M. de Jonge, Phys. Rev. B 65, 064440 (2002).

${ }^{9}$ M. Schleberger, P. Walser, M. Hunziker, and M. Landolt, Phys.
Rev. B 60, 14360 (1999).

${ }^{10}$ G. S. Patrin, S. G. Ovchinnikov, D. A. Velikanov, and V. P. Kononov, Solid State Phys. 43, 1643 (2001).

${ }^{11}$ C. B. Duke, in Tunneling Phenomena in Solids, edited by E. Burstein and S. Lundquist (Plenum, New York, 1969); M. D. Stiles, in Ultrathin Magnetic Structures III, edited by B. Heinrich and J. A. C. Bland (Springer, New York, 2005).

${ }^{12}$ Y. P. Lee, C. O. Kim, J. Y. Baek, K. W. Kim, J. Y. Rheec, Y. V. Kudryavtsev, and J. Dubowik, J. Magn. Magn. Mater. 226-230, 1790 (2001).

${ }^{13}$ Y. Endo, O. Kitakami, and Y. Shimada, Phys. Rev. B 59, 4279 (1999).

${ }^{14}$ R. R. Gareev, D. E. Bürgler, M. Buchmeier, D. Olligs, R. Schreiber, and P. Grünberg, Phys. Rev. Lett. 87, 157202 (2001).

${ }^{15}$ R. R. Gareev, D. E. Bürgler, M. Buchmeier, R. Schreiber, and P. Grünberg, J. Magn. Magn. Mater. 240, 235 (2002).

${ }^{16}$ B. Croonenborghs, F. M. Almeida, C. L'abbe, R. R. Gareev, M. Rots, A. Vantomme, and J. Meersschaut, Phys. Rev. B 71, 024410 (2005) 
${ }^{17}$ J. C. Slonczewski, Phys. Rev. B 39, 6995 (1989).

${ }^{18}$ P. Bruno, Phys. Rev. B 52, 411 (1995).

${ }^{19}$ K. Xia, W. Zhang, M. Lu, and H. Zhai, Phys. Rev. B 56, 14901 (1997).

${ }^{20}$ J. M. Pruneda, R. Robles, S. Bouarab, J. Ferrer, and A. Vega, Phys. Rev. B 65, 024440 (2002).

${ }^{21}$ P. T. Jochym, K. Parlinski, and A. M. Oles, Phys. Rev. B 73, 224411 (2006).

${ }^{22}$ M. Stiles, J. Magn. Magn. Mater. 200, 322 (1999).

${ }^{23}$ Z.-P. Shi, P. Levy, and J. Fry, Europhys. Lett. 26, 473 (1994).

${ }^{24}$ E. Kulatov, H. Ohta, T. Arioka, S. Halilov, and L. Vinokurova, J. Phys.: Condens. Matter 9, 9043 (1997); E. Kulatov and H. Ohta, J. Phys. Soc. Jpn. 66, 2386 (1997).

${ }^{25}$ A. A. Abrikosov, L. P Gor'kov, and I. E. Dzyaloshinski, Methods of Quantum Field Theory in Statistical Physics (Dover, New York, 1963).

${ }^{26}$ G. L. Bir and G. E. Picus, Symmetry and Strain-Induced Effects in Semiconductors (Nauka, Moscow, 1972).

${ }^{27}$ J. C. Slonczewski, Phys. Rev. Lett. 67, 3172 (1991).
${ }^{28}$ T. Moriya, Spin Fluctuation in Itinerant Electron Magnetism (Springer, Berlin, 1985).

${ }^{29}$ V. Tugushev, E. Kulatov, and V. Men'shov, Physica B 378-380, 1100 (2006).

${ }^{30}$ J. C. Slonczewski, J. Appl. Phys. 73, 5957 (1993).

${ }^{31}$ J. M. Fallon, C. A. Faunce, H. J. Blythe, and P. J. Grundy, J. Magn. Magn. Mater. 198-199, 728 (1999).

${ }^{32}$ J. M. Fallon, C. A. Faunce, and P. J. Grundy, J. Appl. Phys. 87, 6833 (2000); J. Phys.: Condens. Matter 12, 4075 (2000); J. Appl. Phys. 88, 2400 (2000).

${ }^{33}$ P. J. Grundy, J. M. Fallon, and H. J. Blythe, Phys. Rev. B 62, 9566 (2000).

${ }^{34}$ K. Inomata, and Y. Saito, J. Appl. Phys. 81, 5344 (1997).

${ }^{35}$ C. Quirós, J. I. Martín, L. Zárate, M. Vélez, and J. M. Alameda, Phys. Rev. B 71, 024423 (2005).

${ }^{36}$ V. Barzykin, Phys. Rev. B 71, 155203 (2005).

${ }^{37}$ V. Tugushev, E. Kulatov, O. Navarro, Microelectron. J. 36, 472 (2005). 